\title{
Erratum to: Mammographic texture and risk of breast cancer by tumor type and estrogen receptor status
}

\author{
Serghei Malkov ${ }^{1 *}$, John A. Shepherd ${ }^{1}$, Christopher G. Scott ${ }^{2}$, Rulla M. Tamimi ${ }^{4}$, Lin Ma ${ }^{3}$, Kimberly A. Bertrand ${ }^{5}$, \\ Fergus Couch², Matthew R. Jensen², Amir P. Mahmoudzadeh', Bo Fan', Aaron Norman², Kathleen R. Brandt' \\ V. Shane Pankratz ${ }^{2}$, Celine M. Vachon ${ }^{2+}$ and Karla Kerlikowske ${ }^{3 \dagger}$
}

\section{Erratum}

After publication of the original article [1], the author noticed some errors to the article [1] which are included in this erratum. All errors reported in this erratum have been updated in the original article [1].

In the 'Results' section of the article Abstract, the sentence "Entropy was associated with a decreased risk of breast cancer" should be "Entropy was associated with an increased risk of breast cancer". In the same section, the variables "FD_TH10", "FD_TH15", "FD_TH60", "FD_TH85", "FD_TH75", and "FD_TH75" should include an additional underscore so they are presented as: "FD_TH_10", "FD_TH_15", "FD_TH_60", "FD_TH_85", "FD_TH_75", and "FD_TH_75", respectively. All variables should be italicized.

In the section entitled 'Breast texture measurements', the sentence "the image with only constant grayscale pixels has Energy equal to 0" should state "the image with only constant grayscale pixels has Energy equal to 1".

In the 'Results' section of the article [1], the variable "FD_TH_75" included in sentence: "The top left and bottom left images show a top 20th percent tile value of FD_TH_75" should be included in italics.

In the 'Abbreviations' section, "BGTDM" should be "NGTDM". The correct version of the 'Abbreviations' is included in this erratum and has been updated in the original article [1].

In Table 1, in the column "Texture feature name", the names of variables should be in italics. In addition in Table 5, in the column "Feature", all variables should also be included in italics. The revised versions of Tables 1 and 5 are included in this erratum and have been updated in the original article [1].

\section{Abbreviations}

AUC: Area under the curve; BI-RADS: Breast Imaging-Reporting and Data System; BMI: Body mass index; cc: Craniocaudal; Cl: Confidence interval; DCIS: Ductal carcinoma in situ; ER: Estrogen receptor; FD: fractal dimension; FT: Fourier transform; GLCM: Gray-level co-occurrence matrix; MCMAM: Mayo Clinic Mammography Study; MMHS: Mayo Mammography Health Study; NGTDM: Neighborhood gray-tone difference matrix; NHS: Nurses' Health Study; OR: Odds ratio; PD: Percent density; SD: Standard deviation; SFMR: San Francisco Bay Area Breast Cancer SPORE and San Francisco Mammography Registry; UCSF: University of California San Francisco

\section{Author details}

${ }^{1}$ Department of Radiology and Biomedical Imaging, UCSF School of Medicine, San Francisco, CA, USA. ${ }^{2}$ Mayo Clinic, Rochester, MN, USA. ${ }^{3}$ UCSF Departments of Medicine and Epidemiology/Biostatistics, San Francisco, CA, USA. ${ }^{4}$ Harvard Medical School, Boston, MA, USA. ${ }^{5}$ Slone Epidemiology Center at Boston University, Boston, MA, USA.

Received: 14 December 2016 Accepted: 14 December 2016 Published online: 04 January 2017

\section{References}

1. Malkov S, et al. Mammographic texture and risk of breast cancer by tumor type and estrogen receptor status. Breast Cancer Research. 2016;18:122. doi:10.1186/s13058-016-0778-1.

* Correspondence: serghei.malkov@ucsf.edu

${ }^{\dagger}$ Equal contributors

${ }^{1}$ Department of Radiology and Biomedical Imaging, UCSF School of

Medicine, San Francisco, CA, USA

Full list of author information is available at the end of the article 
Table 1 Image texture features that are currently defined for all study participants

\begin{tabular}{|c|c|c|c|}
\hline Analysis Groups & Texture Features & Texture feature name & Reference \\
\hline \multirow[t]{4}{*}{ Gray-level Histogram } & Standard deviation & STD & \multirow[t]{4}{*}[7,22,24-26]{} \\
\hline & Skewness & Skewness & \\
\hline & Kurtosis & Kurtosis & \\
\hline & Balance & Balance & \\
\hline \multirow[t]{8}{*}{ Grey-level co-occurrence matrix (GLCM) } & GLCM Energy & Energy & \multirow[t]{8}{*}[24,25,27,29]{} \\
\hline & GLCM Entropy & Entropy & \\
\hline & GLCM Dissimilarity & Dissimilarity & \\
\hline & GLCM Contrast & Contrast & \\
\hline & GLCM Homogeneity & Homogeneity & \\
\hline & GLCM Correlation & Correlation & \\
\hline & GLCM Mean & GLCM Mean & \\
\hline & GLCM Variance & GLCM Variance & \\
\hline \multirow[t]{5}{*}{ Neighborhood Gray-tone Difference Matrix (NGTDM) } & NGTDM Coarseness & NGTDM Coarseness & \multirow[t]{5}{*}[24,28,29]{} \\
\hline & NGTDM Contrast & NGTDM Contrast & \\
\hline & NGTDM Complexity & Complexity & \\
\hline & NGTDM Strength & Strength & \\
\hline & NGTDM Busyness & Busyness & \\
\hline Edge Frequency Analysis & Mean Gradient & Mean_Gradient & [29] \\
\hline \multirow[t]{4}{*}{ Fourier Transform Analysis, Power Spectrum } & RMS & FT_RMS & \multirow[t]{4}{*}{ [29] } \\
\hline & FMP (first moment of power spectrum) & FT_FMP & \\
\hline & SMP (second moment of power spectrum) & FT_SMP & \\
\hline & FD from power spectrum exponent & FT_FD & \\
\hline \multirow[t]{7}{*}{ Fractal Analysis } & $\begin{array}{l}\text { Intercept of the plot of the standard deviation of the high } \\
\text { frequency image as a function of the size the kernel }\end{array}$ & CD_Yint & \multirow[t]{7}{*}{ [29-31] } \\
\hline & Continuous Dimension (CD), slope and intercept & CD_Slope & \\
\hline & HZ_PROJ & $H Z \_P R O J$ & \\
\hline & FD of the standard deviation & FD_Sigma & \\
\hline & FD of image using thresholds from 5\%-85\% & FD_TH_5: FD_TH_85 & \\
\hline & $\begin{array}{l}\text { FD of the surface of thebreast considering the gray } \\
\text { value represent the height }\end{array}$ & FD_CALDWELL & \\
\hline & FD, Minkowski method & FD_Minkowski & \\
\hline
\end{tabular}


Table 5 Risk associated of either DCIS or Invasive Cancer for each feature

\begin{tabular}{|c|c|c|c|c|c|c|c|c|}
\hline \multirow[t]{2}{*}{ Feature } & DCIS & Invasive & & & ER- & ER+ & & \\
\hline & OR (95\% Cl) & OR $(95 \% \mathrm{Cl})$ & p-value* & p-het ${ }^{* *}$ & OR (95\% Cl) & OR $(95 \% \mathrm{Cl})$ & $p$-value * & p-het ${ }^{* *}$ \\
\hline $\mathrm{N}$ case/control & $254 / 1659$ & $908 / 1659$ & & & $116 / 1291$ & $746 / 1291$ & & \\
\hline FD_TH_75 & $0.87(0.74,1.01)$ & $0.87(0.78,0.96)$ & 0.010 & 0.98 & $0.84(0.67,1.06)$ & $0.88(0.79,0.99)$ & 0.048 & 0.72 \\
\hline Energy & $0.88(0.76,1.02)$ & $0.88(0.80,0.96)$ & 0.011 & 0.93 & $0.85(0.69,1.05)$ & $0.86(0.78,0.95)$ & 0.009 & 0.90 \\
\hline Entropy & $1.18(1.02,1.38)$ & $1.13(1.03,1.25)$ & 0.010 & 0.60 & $1.16(0.93,1.44)$ & $1.15(1.03,1.28)$ & 0.024 & 0.96 \\
\hline FD_TH_70 & $0.85(0.72,1.00)$ & $0.87(0.79,0.97)$ & 0.015 & 0.75 & $0.84(0.67,1.06)$ & $0.89(0.79,1.00)$ & 0.085 & 0.64 \\
\hline FD_TH_80 & $0.90(0.77,1.04)$ & $0.89(0.81,0.98)$ & 0.034 & 0.90 & $0.85(0.68,1.05)$ & $0.89(0.80,1.00)$ & 0.066 & 0.64 \\
\hline FD_TH_10 & $1.19(1.04,1.38)$ & $1.09(0.99,1.19)$ & 0.022 & 0.21 & $1.03(0.84,1.26)$ & $1.06(0.96,1.18)$ & 0.479 & 0.75 \\
\hline Kurtosis & $0.86(0.73,1.00)$ & $0.90(0.81,0.99)$ & 0.032 & 0.58 & $0.98(0.78,1.22)$ & $0.91(0.81,1.01)$ & 0.216 & 0.53 \\
\hline FD_TH_65 & $0.84(0.71,0.99)$ & $0.89(0.80,0.99)$ & 0.035 & 0.49 & $0.83(0.65,1.06)$ & $0.91(0.81,1.03)$ & 0.170 & 0.46 \\
\hline FD_Minkowski & $0.90(0.74,1.08)$ & $0.86(0.77,0.97)$ & 0.042 & 0.71 & $0.77(0.59,1.01)$ & $0.89(0.78,1.01)$ & 0.063 & 0.32 \\
\hline Busyness & $1.15(1.00,1.33)$ & $1.09(1.00,1.19)$ & 0.053 & 0.46 & $0.92(0.75,1.14)$ & $1.09(0.99,1.21)$ & 0.128 & 0.12 \\
\hline Homogeneity & $1.05(0.90,1.22)$ & $1.13(1.03,1.24)$ & 0.042 & 0.36 & $1.06(0.86,1.31)$ & $1.12(1.01,1.24)$ & 0.091 & 0.62 \\
\hline Dissimilarity & $0.96(0.83,1.12)$ & $0.89(0.81,0.98)$ & 0.057 & 0.35 & $0.95(0.77,1.17)$ & $0.89(0.81,0.99)$ & 0.110 & 0.61 \\
\hline FD_TH_60 & $0.85(0.71,1.02)$ & $0.9(0.8,1.01)$ & 0.077 & 0.56 & $0.9(0.69,1.15)$ & $0.92(0.81,1.04)$ & 0.348 & 0.85 \\
\hline FD_TH_85 & $0.92(0.79,1.06)$ & $0.91(0.83,1)$ & 0.130 & 0.98 & $0.89(0.72,1.09)$ & $0.91(0.82,1.01)$ & 0.173 & 0.77 \\
\hline FD_TH_15 & $1.2(1.04,1.39)$ & $1.06(0.97,1.16)$ & 0.034 & 0.09 & $0.96(0.78,1.18)$ & $1.05(0.95,1.16)$ & 0.572 & 0.43 \\
\hline
\end{tabular}

Results presented as OR per 1 SD in normalized feature after adjustment for age, family history, PD, and study

${ }^{*} p$-value refers to 2 degree of freedom to test for evidence of associated with DCIS or invasive cancer

**Heterogeneity $\mathrm{p}$-value to test for differences in effect between tumor subgroups 